\title{
Risk Factors for Herpes Zoster: a Systematic Review and Meta-Analysis
}

\section{Citation}

Kawai, Kosuke, and Barbara P Yawn. 2017. “Risk Factors for Herpes Zoster: a Systematic Review and Meta-Analysis." Open Forum Infectious Diseases 4 (Suppl 1): S313-S314. doi:10.1093/ofid/ ofx163.733. http://dx.doi.org/10.1093/ofid/ofx163.733.

\section{Published Version}

doi:10.1093/ofid/ofx163.733

\section{Permanent link}

http://nrs.harvard.edu/urn-3:HUL.InstRepos:34493190

\section{Terms of Use}

This article was downloaded from Harvard University's DASH repository, and is made available under the terms and conditions applicable to Other Posted Material, as set forth at http:// nrs.harvard.edu/urn-3:HUL.InstRepos:dash.current.terms-of-use\#LAA

\section{Share Your Story}

The Harvard community has made this article openly available.

Please share how this access benefits you. Submit a story.

\section{Accessibility}


Turkey, ${ }^{3}$ Department of Infectious Diseases and Clinical Microbiology, Recep Tayyip Erdogan University, Rize, Turkey, ${ }^{4}$ Department of Infectious Diseases and Clinical Microbiology, Amasya University Sabuncuoglu Serefeddin Training and Research Hospital, Amasya, Turkey, ${ }^{5}$ Department of Medical Biochemistry, Karadeniz Technical University, Faculty of Medicine, Trabzon, Turkey

Session: 139. Adult Viral Infection

Friday, October 6, 2017: 12:30 PM

Background. Crimean-Congo hemorrhagic fever (CCHF) is a potentially fatal disease caused by a tick-borne virus from the Bunyaviridae family. Cytokines plays an important role in the pathogenesis of viral, bacterial, and immunologic diseases. This study aimed to investigate the role of TNF-alpha, IL-6, IL-10, and IFN-gamma levels in the severity of infection and clinical outcome of patients with CCHF.

Methods. Patients with confirmed CCHF were divided into two groups (severe cases: Patients who exhibited hemorrhage during their hospital stay, and mild/ moderate cases: Patients who displayed no hemorrhage during their hospital stay). Demographic characteristics, laboratory tests on admission of all patients with CCHF were investigated, and serum TNF-alpha, IL-6, IL-10, and IFN-gamma levels were measured.

Results. A total of 154 patients with confirmed CCHF were investigated. Fortysix $(29.9 \%)$ of these patients were in the severe group. In patients with severe CCHF significantly higher serum levels of TNF-alpha $(68.2 \pm 23.5 ; P=0.008)$ and IL-6 $(73.1 \pm 41.6 ; P=0.003)$ were detected, compared with cytokine levels in patients who mild/moderate CCHF (Table 1). No differences in serum IL-10 and IFN-gamma levels between patients who severe CCHF and those who mild/moderate CCHF were detected $(P>0.05)$

Table 1: Cytokine levels, demographic and laboratory characteristics in patients with severe and mild/moderate cases with CCHF.

\begin{tabular}{lccc}
\hline Features & $\begin{array}{c}\text { Severe cases } \\
n=46\end{array}$ & $\begin{array}{c}\text { Mild/moderate cases } \\
n=108\end{array}$ & P-value \\
\hline Age & $50.6 \pm 20.3$ & $49.8 \pm 21.0$ & 0.682 \\
Female gender, & $31(67.4)$ & $63(58,3)$ & 0.291 \\
$\quad n(\%)$ & & \\
TNF- & $68.2 \pm 23.5$ & $41.3 \pm 17.4$ & 0.008 \\
IL-6 & $73.1 \pm 41.6$ & $38.0 \pm 19.5$ & 0.003 \\
IL-10 & $6.32 \pm 1.3$ & $6.21 \pm 1.4$ & 0.753 \\
IFN-gamma & $145 \pm 96$ & $126 \pm 92$ & 0.664 \\
WBC & $3286 \pm 5,602$ & $2,275 \pm 1,286$ & 0.280 \\
PLT & $53,564 \pm 36,520$ & $98,065 \pm 42,768$ & 0.001 \\
CRP & $3.2 \pm 2.6$ & $1.1 \pm 1.4$ & 0.005 \\
ALT & $521 \pm 482$ & $208 \pm 320$ & 0.044 \\
AST & $869 \pm 1.182$ & $256 \pm 215$ & 0.016 \\
CPK & $1,138 \pm 970$ & $676 \pm 835$ & 0.007 \\
LDH & $1,800 \pm 1,254$ & $589 \pm 271$ & 0.002 \\
\hline
\end{tabular}

Conclusion. Cytokines, chemokines, and other inflammatory mediators function in a manner, acting on many different cell types to regulate the host's immune response. When cytokines present in high concentrations, they might toxic or even lethal effects. In accordance with this view, we have detected increased serum TNF-alpha, IL-6 levels in the patients with severe $\mathrm{CCHF}$

Disclosures. All authors: No reported disclosures.

\section{Etiologic Involvement of Enterovirus and Human Bocavirus in Acute} Flaccid Paralysis Cases in India

Manjari Baluni, Ph.D(Pursuing) ${ }^{1}$; Dharamveer Singh, Ph.D(Pursuing) ${ }^{1}$;

Sneha Ghildiyal, PhD Pursuing ${ }^{2}$; Tanzeem Fatima, Ph.D (Pursuing) ${ }^{3}$; Amreen Zia, $\mathrm{Ph} . \mathrm{D}$ (Pursuing) ${ }^{3}$ and Tapan Dhole, $\mathrm{MD}^{1}{ }^{1}{ }^{1}$ Sanjay Gandhi Post Graduate Institute of Medical Sciences, Lucknow, India, ${ }^{2}$ Sanjay Gandhi Postgraduate Institute of Medical Sciences, Lucknow, India, ${ }^{3}$ Microbiology, Sanjay Gandhi Post Graduate Institute of Medical Sciences, Lucknow, India

Session: 139. Adult Viral Infection

Friday, October 6, 2017: 12:30 PM

Background. Acute flaccid paralysis (AFP), characterized by the rapid onset of asymmetric paralysis, can be caused by a variety of viral infections or coinfections. Besides wild-type and revertant vaccine strains of polioviruses, several nonpolio enteroviruses, have also been associated with AFP. Enteroviruses (EVs) are RNA viruses in the family Picornaviridae comprising more than 100 serotypes that are divided into four species, human enteroviruses A to D. The clinical manifestations of EVs range from conjunctivitis, respiratory tract infection, myocarditis, meningitis, encephalitis, and neonatal sepsis, like illness. Human Bocavirus (HBoV), a newly classified member of the Parvoviridae family, has been detected frequently in feces of diarrhoeic children suggesting its possible etiological involvement in the disease.

Methods. Total 586 stool specimens were collected in 2016 from children suspected for AFP. Molecular method for targeting 5' untranslated region (UTR) and VP1 capsid region was used for detection of human enteroviruses (HEV), human boca viruses $(\mathrm{HBoV})$ and saffold viruses in direct clinical specimen.
Results. EV RNA was detected in 103 (17.6\%) of 586 stool specimens by real-time RT-PCR targeting the highly conserved 5' UTR region. Out of them, 71 (12.11\%) were NPEV, partially sequenced by VP1 which revealed the prevalence of echovirus (ECV) $19(n=6), \operatorname{ECV} 11(\mathrm{n}=7), \operatorname{ECV} 18(n=4), \operatorname{ECV} 33(n=5), \operatorname{ECV} 29(n=1), \operatorname{ECV} 25$ $(n=2), \operatorname{ECV} 24(n=3), \operatorname{ECV} 3(n=3), \operatorname{ECV} 14(n=2), \operatorname{ECV} 13(n=1), \operatorname{ECV} 2(n=1)$, ECV $20(n=2), \operatorname{ECV} 27(n=4), \operatorname{ECV} 6(n=2), \operatorname{CV} \mathrm{A} 10(n=2), \mathrm{CV}$ A9 $(n=1), \mathrm{CV}$ A6 $(n=2), \mathrm{CV}$ B4 $(n=1), \mathrm{CV}$ B5 $(n=3), \mathrm{CV}$ B6 $(n=3), \mathrm{EV} 80(n=1), \mathrm{EV} 83(n=1)$, EV $97(n=2)$.

Total $63(10.75 \%)$ HBoVs were detected by real-time PCR which were further sequenced by VP1, consists of HBoV-1 $(n=8), \mathrm{HBoV}-2(n=15), \mathrm{HBoV}-3(n=9)$ and HBoV-4 $(n=5)$. Out of them $9(1.5 \%)$ were detected as co infection with NPEVs. Phylogenetic analysis showed 0.9 - 5.6\% divergence at nucleotide level among HBoVs.

Total $9(1.5 \%)$ saffold viruses was detected and characterized by VP1 sequencing.

Conclusion. ECV and $\mathrm{HBoV}$ were found the main etiologic agent in children suspected with AFP. Molecular typing of these viruses is useful for characterizing emerging serotypes and their epidemiological investigation.

Disclosures. All authors: No reported disclosures.

\section{Frequency of Epstein-Barr Virus Genotypes in Pakistani Trangender SexWorkers}

Sadia Salahud Din, Master; Biological Sciences, Gomal University, Dera Islamail Khan, Pakistan

Session: 139. Adult Viral Infection

Friday, October 6, 2017: 12:30 PM

Background. Transgender community large association with sex work has put them at a greater risk of contracting sexually transmitted infections (STIs).The aim of this study was to investigate the prevalence of Epstein-Barr Virus (EBV) genotypes in transgender sex workers (TSWs) of twin-cities of Pakistan. The high prevalence of EBV-2 genotype in sex workers has been previously reported. EBV genotypes were investigated in transgender sex workers to find out EBV-2 occurrence in Pakistani population.

Methods. A total of 86 transgender (Hijras) sex workers were randomly included in this study. Demographics, including age, the number of sex partners, sexual habits, and awareness about protective methods were obtained. Blood was collected from all subjects and The presence of Human Immunodeficiency Virus, Hepatitis B and C virus were determined by antibody strip testing. EBV detection and genotyping were performed by extracting genomic DNA from all whole blood samples. B-globin and EBNA-1 were amplified to assess the quality and presence of EBV DNA. Analysis of EBNA-2 genotyping was done by nested PCR.

Results. HIV was the most prevalent infection in 40 transgender sex workers (46.51\%) followed by HCV in 15 (17.44\%). Among HIV-seropositive TSW's, EBV genotype determination was only achievable in $60 \%$ of cases, where $62.5 \%$ were EBV- 1 , $29.16 \%$ of EBV-2 and co-infection was found in $8 \%$ samples. Among HIV-negative individuals, $78 \%$ were EBV-1, whereas EBV-2 genotype and co-infections were absent. All non-typable samples were amplifiable for the EBNA-1 gene in both populations, confirming EBV genome in the samples.

Conclusion. EBV-1 was the most common genotype of EBV in HIV seropositive and seronegative TSW's but the high occurrence of EBV-2 and co-infection of both types was observed only in HIV seropositive individuals. This is the first report of frequency of EBV infections in the HIV-positive transgender community of Pakistan.

Disclosures. All authors: No reported disclosures.

1036. Risk Factors for Herpes Zoster: a Systematic Review and Meta-Analysis Kosuke Kawai, $\mathrm{ScD}^{1}$ and Barbara P. Yawn, MD, MSc, MSPH, FAAFP ${ }^{2} ;{ }^{1}$ Boston Children's Hospital and Ḧarvard Medical School, Boston, Massachusetts, ${ }^{2}$ Department of Research, Olmsted Medical Center, Rochester, Minnesota; University of Minnesota, Minneapolis, Minnesota

Session: 139. Adult Viral Infection

Friday, October 6, 2017: 12:30 PM

Background. Well-recognized risk factors for herpes zoster (HZ), commonly known as shingles, are age and immunosuppression. Numerous studies have investigated other various risk factors for $\mathrm{HZ}$ in recent years. The objective of our study is to systematically review studies examining risk factors for $\mathrm{HZ}$ and discuss implications based on the updated evidence.

Methods. We performed a literature search using PubMed, Embase, and Web of Science and included studies that examined risk factors for HZ. Random effects model was used to summarize the risk ratio (RR) or odds ratio (OR) and $95 \%$ confidence interval (CI).

Results. Of the 3450 studies screened, we included 84 studies in the systematic review and conducted meta-analysis in 62 studies. Women are at increased risk of $\mathrm{HZ}$ compared with men (pooled adjusted $\mathrm{RR}=1.31$; $95 \% \mathrm{CI}: 1.27,1.34$ ). Black individuals have almost half the risk of $\mathrm{HZ}$ than White individuals (pooled $\mathrm{RR}=0.54$ 95\% CI: $0.47,0.63$ ). Family history was found to be a risk factor for $\mathrm{HZ}$ (pooled $\mathrm{OR}=3.59 ; 95 \% \mathrm{CI}: 2.39,5.40)$. Autoimmune diseases, including rheumatoid arthritis (pooled RR $=1.67 ; 95 \%$ CI: $1.41,1.98)$ and systemic lupus erythematous (RR $=2.10$; $95 \%$ CI: $1.40,3.15$ ), were associated with an elevated risk of HZ. Other comorbidities were associated with an increased risk of $\mathrm{HZ}$, with the pooled RRs ranging from 1.25 (95\% CI: $1.13,1.39)$ for asthma to $1.30(1.17,1.45)$ for diabetes mellitus, and 1.31 (95\% CI: $1.22,1.41)$ for chronic obstructive pulmonary disease. Statin use was also 
associated with a modest increased risk of $\mathrm{HZ}$ (pooled RR $=1.14 ; 95 \%$ CI: 1.11, 1.17) Recent physical trauma increased risk of HZ by almost two-fold (pooled RR $=2.56$ 95\% CI: 1.97, 3.33).

Conclusion. In addition to age and immunocompromised conditions, our review shows that female sex, race/ethnicity, family history, and comorbidities are risk factors for HZ. Efforts are needed to better understand risk factors and to increase the uptake of zoster vaccination.

Disclosures. B. P. Yawn, GSK: Consultant and Scientific Advisor, Consulting fee

\section{Herpes-Zoster Infection in a Tertiary Hospital in Brazil}

Luciana Antoniolli, Medical student ${ }^{1}$; Aline Azambuja, $\mathrm{MD}, \mathrm{PhD}^{2}$; Camila Rodrigues Medical student ${ }^{1} ;$ Rafael Borges, medical student ${ }^{1}$ and Luciano Goldani, PhD, MD

${ }^{1}$ Federal University of Rio Grande do Sul, Porto Alegre, Brazil, ${ }^{2}$ Infectious Diseases, Federal University of Rio Grande do Sul, Porto Alegre, Brazil, ${ }^{3}$ Internal Medicine, Infectious Diseases Unit, Hospital de Clinicas de Porto Alegre, Porto Alegre, Brazil

Session: 139. Adult Viral Infection

Friday, October 6, 2017: 12:30 PM

Background. herpes zoster (HZ) is a common infection with potential com plications requiring hospital care, especially for patients with multiple comorbities. However, there is little information on $\mathrm{HZ}$ from hospital registries.

Methods. we searched for hospital-based records of B02 code (ICD-10) between March 2000 and January 2017 at Hospital de Clínicas de Porto Alegre, a tertiary, university hospital in south Brazil. To avoid misclassifications, we considered clinica evaluation for the diagnosis of cutaneous $\mathrm{HZ}$ and postherpetic neuralgia (PHN), ophthalmological evaluation for ophthalmic $\mathrm{HZ}$ and the combination of clinical, radiologic and cerebrospinal fluid analysis for $\mathrm{HZ}$ meningo-encephalitis (ME). We analyzed conditions associated with immune dysregulation, complications, length of hospita stay, and mortality. Chi-square test and Kaplan-Meier estimator were used for statistical analyses. $P<0.05$ was considered statistically significant.

Results. there were 847 records for this period, of which 801 were confirmed according to our criteria and included in the analysis. Most patients were women ( $n=$ $448 ; 60 \%$ ), with an average of 48.8 years, standard deviation of 22.2 . There were more diagnoses in the inpatients group (74.4\%), and fewer in the emergency room $(22.4 \%)$ and outpatient (3.3\%). The median length of hospital stay was 7 days (2-10, P25-P75) when $\mathrm{HZ}$ was the main reason for admission. Most patients presented cutaneous HZ $(n=743,92.8 \%)$. There were fewer cases of PHN (6.1\%), ophthalmic HZ (7.6\%) and ME (4.1\%). Seventy percent had some kind of immune dysregulation; more frequently AIDS (31\%), use of immunosuppressive agents (18.7\%) and malignant disease (16.2\%). We followed the subjects for a median of 28.2 (2.8-77.5) months. During this period, there were $105(13.1 \%)$ deaths. Five were related to HZ ME. The 30-day overall mortality rate was $1.5 \%$. There was no statistical difference in cumulative survival (graph $1, P=0.05$ ) or incidence of complicated forms for patients with or without immune dysregulation.

Conclusion. our sample was characterized by a majority of inpatient diagnoses. The 30-day mortality rate was lower than reported in similar studies, but there was a relevant impact of complicated forms in mortality and sequelae.

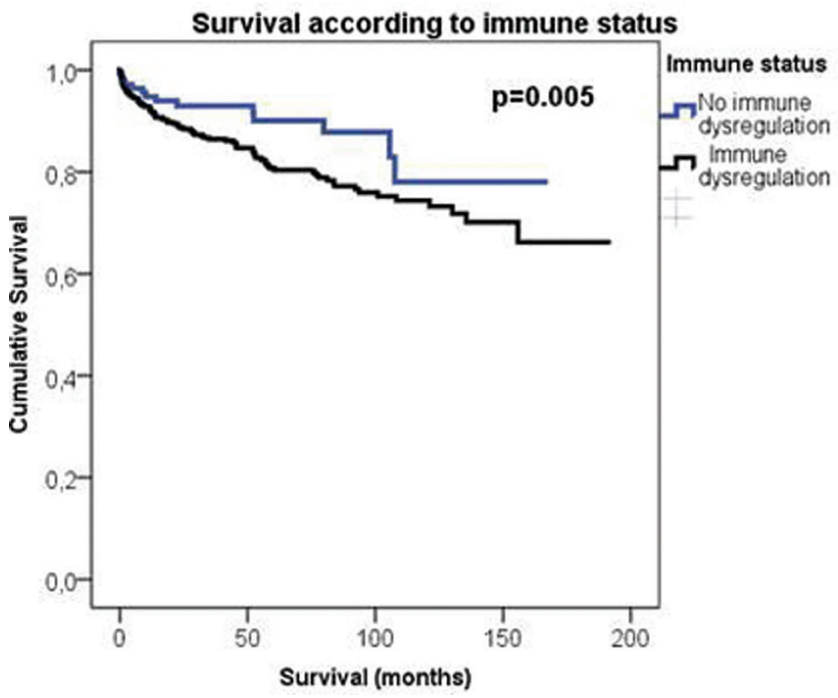

Disclosures. All authors: No reported disclosures.

1038. Epidemiology of Influenza Viruses in Canada over the 2011-2012 to 20132014 Seasons: A Study from the Serious Outcomes Surveillance (SOS) Network of the Canadian Immunization Research Network (CIRN)

May Elsherif, $\mathrm{MD}^{1}$; Todd Hatchette, MD FRCPC ${ }^{2}$; Jason Leblanc, $\mathrm{PhD}^{1}$; Lingyun Ye, MSc ${ }^{1}$; Melissa K Andrew, MD, $\mathrm{PhD}^{1}$; Ardith Ambrose, $\mathrm{RN}^{1}$; Guy Boivin, $\mathrm{MD}, \mathrm{MSc}^{3}$;
William R. Bowie, MD, FRCPC, FIDSA ${ }^{4}$; Karen Green, MSc, RN ${ }^{5}$; Kevin Katz, MD CM, MSc, FRCPC ${ }^{6}$; Mark Loeb, MD, MSc ${ }^{7}$; Donna Mackinnon-Cameron, MMath ${ }^{1}$ Anne Mccarthy, MD, MSc ${ }^{8}$; Janet McElhaney, $\mathrm{MD}^{9}$; Allison Mcgeer, MD, MSc ${ }^{5}$; Michaela Nichols, $\mathrm{MSc}^{1}$; Jeff Powis, MD, MSc, FRCPC ${ }^{10}$; David Richardson, MD $^{11}$; Makeda Semret, $\mathrm{MD}^{12}$; Daniel Smyth, MD, FRCPC ${ }^{13}$; Sylvie Trottier, MD, $\mathrm{PhD}^{3}$; Louis Valiquette, MD, MSc, FRCPC ${ }^{14}$; Duncan Webster, MD $^{15}$ and Shelly McNeil, MD, FRCPC, FIDSA ${ }^{1}$; ${ }^{1}$ Canadian Center for Vaccinology, IWK Health Centre and Nova Scotia Health Authority, Dalhousie University, Halifax, NS, Canada, ${ }^{2}$ Nova Scotia Health Authority, Dalhousie University, Halifax, NS, Canada, ${ }^{3}$ Centre Hospitalier Universitaire de Quebec, Quebec City, QC, Canada, ${ }^{4}$ Division of Infectious Diseases, Department of Medicine, University of British Columbia, Vancouver, BC, Canada, ${ }^{5}$ Mount Sinai Hospital, Toronto, ON, Canada, ${ }^{6}$ North York General Hospital, Toronto, ON, Canada, ${ }^{7}$ McMaster University, Hamilton, ON, Canada, ${ }^{8}$ The Ottawa Hospital, Ottawa, ON, Canada, ${ }^{9}$ Health Sciences North Research Institute, Sudbury, ON, Canada, ${ }^{10}$ Michael Garron Hospital, Toronto, ON, Canada, ${ }^{11}$ William Osler Health System, Brampton, ON, Canada, ${ }^{12}$ McGill University, Montreal, QC, Canada, ${ }^{13}$ The Moncton Hospital, Moncton, NB, Canada ${ }^{14}$ Microbiology and Infectious Disease, Université de Sherbrooke, Sherbrooke, QC, Canada, ${ }^{15}$ Saint John Regional Hospital, Dalhousie University, Saint John, NB, Canada

Session: 139. Adult Viral Infection

Friday, October 6, 2017: 12:30 PM

Background. Influenza virus activity varies seasonally and within season. Epidemiology of serious influenza outcomes is contingent on the prevalent circulating strain/s and susceptible age group/s. Given the strain variability over the 2011-2012 through 2013-2014 seasons in Canada, this study examined the clinical and epidemiological profiles of different influenza strains causing adult hospitalizations.

Methods. During these three influenza seasons, the Serious Outcomes Surveillance (SOS) Network of the Canadian Immunization Research Network (CIRN) enrolled adults hospitalized with acute respiratory illness across Canada. Nasopharyngeal swabs (NPs) from influenza cases were tested for strain character ization using real-time reverse transcriptase polymerase chain reaction (rtRT-PCR) A primary assay differentiated A and B influenza viruses. Subsequently, influenza A viruses were subtyped as $\mathrm{H} 1 \mathrm{~N} 1$ or $\mathrm{H} 3 \mathrm{~N} 2$, and influenza B lineages were differen tiated as Victoria or Yamagata. Laboratory results were compared with patient demographic data and clinical outcomes.

Results. Over three consecutive influenza seasons, 3394 cases of hospitalized acute respiratory illness were laboratory-confirmed as influenza. At $72.4 \%$, influenza A was predominant across all seasons, while influenza B caused $27.6 \%$. Most of the influenza A cases were due to $\mathrm{H} 3 \mathrm{~N} 2$ (58.7\%), while H1N1 accounted for $41.3 \%$. For influenza B, the Yamagata lineage was predominant at $88.4 \%$ whereas the Victoria lineage accounted for $11.6 \%$. Outcome analyses are presented for each influenza A subtype and influenza B lineage, overall and per season. Considering serious outcomes in patients $\geq 65$, higher proportions of patients hospitalized with the H1N1 strain experienced intensive care unit (ICU) admission and need for mechanical ventilation, while higher proportions of patients hospitalized with B/Yamagata and $\mathrm{H} 3 \mathrm{~N} 2$ died within 30 days of admission.

Conclusion. Comprehensive collection of surveillance data paired with NP specimens by the CIRN SOS Network was conducive to broader understanding of influenza strain activity and associated outcomes at the subtype and lineage level. This data is important to make informed recommendations for the use of multicomponent influenza vaccines.

Disclosures. M. Elsherif, Canadian Institutes of Health Research: Investigator Research grant. Public Health Agency of Canada: Investigator, Research grant GSK: Investigator, Research grant. T. Hatchette, GSK: Grant Investigator, Grant recipient; Pfizer: Grant Investigator, Grant recipient. Abbvie: Speaker for a talk on biologics and risk of TB reactivation, Speaker honorarium.M. K. Andrew, GSK Grant Investigator, Research grant. Pfizer: Grant Investigator, Research grant. Sanofi-Pasteur: Grant Investigator, Research grant. J. McElhaney, GSK Vaccines: Scientific Advisor, Speaker honorarium. A. Mcgeer, Hoffman La Roche: Investigator, Research grant. GSK: Investigator, Research grant. sanofi pasteur: Investigator, Research grant. J. Powis, Merck: Grant Investigator, Research grant. GSK: Grant Investigator, Research grant. Roche: Grant Investigator, Research grant. Synthetic Biologicals: Investigator, Research grant. M. Semret, GSK: Investigator, Research grant. Pfizer: Investigator, Research grant. S. Trottier, Canadian Institutes of Health Research: Investigator, Research grant. L. Valiquette, GSK: Investigator, Research grant. S. McNeil, GSK: Contract Clinical Trials and Grant Investigator, Research grant. Merck: Contract Clinical Trials and Speaker's Bureau, Speaker honorarium. Novartis: Contract Clinical Trials, No personal renumeration. sanofi pasteur: Contract Clinical Trials, No personal renumeration

1039. Co-circulation of Influenza A and B During the 2016-2017 Influenza Season at Rush University Medical Center Andrew Simms, $\mathrm{MD}^{1}$; Hemil Gonzalez, $\mathrm{MD}^{2}$; Nicholas M. Moore, $\mathrm{MS}^{3}$; Leslie A. Chapman, BS ${ }^{4}$; Karen Lolans, $\mathrm{BS}^{5}$ and Gordon M. Trenholme, $\mathrm{MD}^{6} ;{ }^{1}$ Infectious Disease, Rush University Medical Center, Chicago, Illinois, ${ }^{2}$ Infectious Diseases, Rush University Medical Center, Chicago, Illinois, ${ }^{3}$ Medical Laboratory Science, Rush University Medical Center, Chicago, Illinois, ${ }^{4}$ Illinois Department of Public Health Laboratories, Chicago, Illinois, ${ }^{5}$ Rush University Medical Center, Chicago, Illinois, ${ }^{6}$ Rush University Medical Center; Division of Infectious Diseases, Chicago, Illinois 